\title{
Management of diabetic ketoacidosis
}

MW Savage

\section{Introduction}

Diabetic ketoacidosis (DKA) is the metabolic abnormality of type 1 diabetes. Near patient testing for the ketones is now readily available for monitoring allowing for a shift away from using glucose levels to drive treatment decisions in the management of DKA. National UK guidelines have been developed to reflect the development in technology. ${ }^{1}$ They are evidence based where possible but are also drawn from accumulated multiprofessional knowledge and consensus agreement.

\section{Pathophysiology and diagnosis}

DKA usually occurs as a consequence of insulin deficiency. This enhances hepatic glucose production. Enhanced fat breakdown increases serum free fatty acids that are then metabolised producing large quantities of ketones and metabolic acidosis. Fluid depletion is a serious problem caused by osmotic diuresis. Electrolyte shifts and depletion are, in part, related to the osmotic diuresis. Hyper- and hypokalaemia are particular dangers.

Absolute diagnostic criteria do not exist, however, the following are proposed: ketonaemia $\geqslant 3 \mathrm{mmol} / \mathrm{l}$ by ketone meter, or significant ketonuria (more than $2+$ on standard urine sticks); blood glucose over $11 \mathrm{mmol} / \mathrm{l}$ or known diabetes mellitus; and venous bicarbonate $\left(\mathrm{HCO}_{3}-\right)<15 \mathrm{mmol} / \mathrm{l}$ and/or venous $\mathrm{pH}<7.3$.

\section{Developments in management}

Recent developments now allow us to focus on the underlying metabolic abnormality (ketonaemia), which simplifies the treatment of those who present with modest elevation of blood glucose but with acidosis secondary to ketonaemia 'euglycaemic diabetic ketoacidosis. ${ }^{2,3}$

Blood glucose is routinely checked at the bedside, but portable ketone meters now also allow bedside measurement of 3-betahydroxybutyrate. ${ }^{4-7}$ The resolution of DKA depends upon the suppression of ketonaemia, therefore the measurement of blood ketones now represents best practice in monitoring the response to treatment. ${ }^{8}$ Access to blood gas and blood electrolyte measurements is now usually available within a few minutes of blood being taken. Therefore glucose, ketones and electrolytes, including bicarbonate and venous $\mathrm{pH}$, should be assessed at, or near, the bedside.

MW Savage, consultant physician in diabetes and endocrinology, Diabetes and Endocrinology Centre, North Manchester Diabetes Centre, Manchester

\section{The involvement of diabetes specialist teams}

Diabetes specialist team (DST) involvement shortens patient stay and improves outcomes. ${ }^{9}$ All patients should be reviewed by a member of the DST prior to discharge, not undertaking this is a governance issue. ${ }^{10}$

\section{General management issues}

There is universal agreement that the most important initial therapeutic intervention is appropriate fluid replacement followed by insulin administration. The main aims of fluid replacement are to:

- restore circulatory volume

- clear ketones

- correct electrolyte imbalance.

In patients with kidney failure or heart failure, as well as the elderly and adolescents, the rate and volume of fluid replacement may need to be modified. The aim of the first few litres of fluid is to correct any hypotension, replenish the intravascular deficit, and counteract the effects of the osmotic diuresis with rectification of electrolyte disturbance.

\section{Assessment of severity}

The presence of one or more of the following may indicate severe DKA and admission to a level 2/high dependency unit environment; insertion of a central line and immediate senior review should be considered:

- blood ketones over $6 \mathrm{mmol} / \mathrm{l}$

- bicarbonate level below $5 \mathrm{mmol} / \mathrm{l}$

- venous/arterial $\mathrm{pH}$ below 7.1

- hypokalaemia on admission (under $3.5 \mathrm{mmol} / \mathrm{l}$ )

- Glasgow Coma Scale less than 12 or abnormal AVPU (Alert, Voice, Pain, Unresponsive) scale

- oxygen saturation below $92 \%$ on air (assuming normal baseline respiratory function)

- $\quad$ systolic blood pressure below $90 \mathrm{mmHg}$

- pulse over 100 or below $60 \mathrm{bpm}$.

\section{Insulin therapy and metabolic treatment targets}

A fixed rate intravenous insulin infusion (FRIVII) calculated on 0.1 units $/ \mathrm{kg}$ infusion is recommended. Patient demographics are changing and patients with DKA are now more likely to be obese or suffering with other insulin-resistant 
states, including pregnancy. This has led to the re-emergence of FRIVII in adults in the USA and international paediatric practice. ${ }^{11-13}$ The FRIVII may need to be adjusted; the recommended targets are:

- reduction of the blood ketone concentration by 0.5 $\mathrm{mmol} / \mathrm{l} /$ hour

- if ketone meter not available, the venous bicarbonate should rise by $3 \mathrm{mmol} / \mathrm{l} /$ hour and capillary blood glucose fall by $3 \mathrm{mmol} / \mathrm{l} /$ hour

- potassium should be maintained between 4.0 and $5.0 \mathrm{mmol} / \mathrm{l}$.

\section{Intravenous glucose concentration}

The management should be focused on clearing ketones as well as normalising blood glucose. It is often necessary to administer an intravenous (iv) infusion of $10 \%$ glucose via an iv pump in order to avoid hypoglycaemia and permit the continuation of a FRIVII to suppress ketogenesis. Introduction of $10 \%$ glucose is recommended when the blood glucose falls below $14 \mathrm{mmol} / \mathrm{l}$. It is important to continue $0.9 \%$ sodium chloride solution concurrently via an iv pump.

\section{Continuation of long-acting insulin analogues}

In the last few years the use of long-acting basal insulin analogues (insulin detemir - Levemir ${ }^{\circledR}$, insulin glargine - Lantus ${ }^{\circledR}$ ) has become widespread. Continuation of long-acting analogues during the initial management of DKA provides background insulin when the iv insulin is discontinued. This avoids rebound hyperglycaemia when iv insulin is stopped and should avoid excess length of stay.

\section{Serious complications of DKA and its treatment}

Hypokalaemia and hyperkalaemia are potentially life-threatening conditions during the management of DKA. There is a risk of acute renal failure associated with severe dehydration and it is therefore recommended that no potassium be prescribed with the initial fluid resuscitation or if the serum potassium level remains above $5.5 \mathrm{mmol} / \mathrm{l}$. However, potassium will almost always fall as the DKA is treated with insulin, thus it is recommended that $0.9 \%$ sodium chloride solution with potassium $40 \mathrm{mmol} / \mathrm{l}$ (ready-mixed) is prescribed as long as the serum potassium level is below $5.5 \mathrm{mmol} / \mathrm{l}$ and the patient is passing urine.

If the serum potassium level falls below $3.5 \mathrm{mmol} / \mathrm{l}$ the potassium regimen needs review. All aspects of potassium use must comply with local and national guidance. ${ }^{14}$ Trusts need to ensure that they have local protocols in place, which allow for the safe administration of concentrated potassium injectables. Other serious complications include cerebral and pulmonary oedema which are discussed in the online document. ${ }^{1}$ Close attention to fluid balance and slower fluid replacement is recommended for younger adults; for children $<18$ years of age use the British Society for Paediatric Endocrinology and Diabetes DKA guidelines which can be found at: www.bsped.org.uk/ professional/guidelines/docs/DKAGuideline.pdf

\section{Hypoglycaemia}

The blood glucose may fall very rapidly as ketoacidosis is corrected and a common mistake is to allow it to drop to hypoglycaemic levels. This may result in a rebound ketosis driven by counter-regulatory hormones. Severe hypoglycaemia is also associated with cardiac arrhythmias, acute brain injury and death. It is partly for this reason that $10 \%$ glucose is recommended.

\section{Conclusion and summary}

DKA is a medical emergency with a significant morbidity and mortality. It is now recommended that FRIVII be used with bedside measurement of metabolic parameters. The DST should always be involved as soon as possible and ideally within 24 hours because this has been demonstrated to be associated with a better patient experience and reduced length of stay.

In the management of diabetic ketoacidosis the following guidance should therefore be followed:

- measure blood ketones, venous (not arterial) pH and bicarbonate and use results as treatment markers

- monitor ketones and glucose using bedside meters, when available and operating within their quality assurance range

- monitor electrolytes on blood gas analysers with intermittent laboratory confirmation

- replace 'sliding scale' insulin with weight-based FRIVII

- involve the DST as soon as possible

- continue long-acting insulin analogues as normal. ${ }^{1}$

\section{References}

1 Joint British Diabetes Societies Inpatient Care Group. The management of diabetic ketoacidosis in adults. London: NHS Diabetes, 2010. www.diabetes.nhs.uk/document.php?o=1336

2 Munro JF, Campbell IW, Mccuish AC, Duncan LJP. Euglycaemic diabetic ketoacidosis. BMJ 1973;2:578-80.

3 Jenkins D, Close CE, Krentz AJ, Nattrass M, Wright AD. Euglycaemic diabetic ketoacidosis: does it exist? Acta Diabetol 1993;30:251-3.

4 Sheikj-Ali M, Karon BS, Basu A et al. Can serum beta-hydroxybutyrate be used to diagnose diabetic ketoacidosis? Diab Care 2008;4:643-7.

5 Bektas F, Eray O, Sari R, Akbas H. Point of care testing of diabetic patients in the emergency department. Endocr Res 2004;30:395-402.

6 Khan ASA, Talbot JA, Tiezen KL et al. Evaluation of a bedside blood ketone sensor: the effects of acidosis, hyperglycaemia and acetoacetate on sensor performance. Diab Med 2004;21:782-5.

7 Wallace TM, Matthews DR. Recent advances in the monitoring and management of diabetic ketoacidosis. QJM 2004;97:773-80.

8 Wiggam MI, O'Kane MJ, Harper R et al. Treatment of diabetic ketoacidosis using normalization of blood 3-hydroxybutyrate concentration as the endpoint of emergency management. A randomized controlled study. Diab Care 1997;20:1347-52. 
9 Cavan DA, Hamilton P, Everett J, Kerr D. Reducing hospital inpatient length of stay for patients with diabetes. Diab Med 2001;18:162-4.

10 Clement S, Braithwaite SS, Magee MF et al. Management of diabetes and hyperglycemia in hospitals. Diab Care 2004;27:553-91.

11 Kitabchi, AE, Umpierrez, GE, Miles, JM, Fisher, JN. Hyperglycemic crises in adult patients with diabetes: a consensus statement from the American Diabetes Association. Diab Care 2009;32:1335.

12 British Society for Paediatric Endocrinology and Diabetes (BSPED) guidelines for the management of DKA. www.bsped.org.uk/professional/guidelines/docs/DKAGuideline.pdf

13 International Society for Pediatric and Adolescent Diabetes. (IPSAD) 2007. www.ispad.org/FileCenter/10-wolfsdorf_Ped_Diab_2007,8.28-43.pdf
14 National Patient Safety Authority. Patient Safety Alert. Potassium solutions: risks to patients from errors occurring during intravenous administration. London: NPSA, 2002.

Address for correspondence: Dr MW Savage, Diabetes and Endocrinology Centre, North Manchester Diabetes Centre, Manchester, M8 5RB.

Email: mark.savage@pat.nhs.uk

\title{
How do I manage a patient with suspected acute pulmonary embolism?
}

\author{
Karen KK Sheares
}

Acute pulmonary embolism (PE) is characterised by obstruction of the pulmonary arterial tree by thrombi commonly originating from the leg or pelvic veins. Although deep vein thrombosis (DVT) and PE are manifestations of the same disease, mortality is higher in those who present with PE. ${ }^{1}$ In 2005, it was estimated that 25,000 people in the UK die of preventable hospital-acquired venous thromboembolism (VTE) every year. Community studies have reported an annual incidence for VTE of 1.4-1.8 per 1,000 with a third presenting with PE. The incidence of VTE rises markedly with age; over the age of 75, the annual incidence reached 1 per 100 population. $^{2}$

Ten per cent of acute PE are immediately fatal, with a further $5 \%$ mortality despite treatment. Perfusion defects resolve in $50 \%$ after one month of treatment, with complete resolution in two-thirds. ${ }^{3}$ There is a two-year cumulative incidence of $3.8 \%$ for chronic thromboembolic pulmonary hypertension due to poor resolution and/or a pulmonary arteriopathy. ${ }^{4}$ Six to $15 \%$ of patients have recurrent thrombosis after anticoagulation particularly in those with permanent risk factors.

\section{Risk factors}

The risk factors for development of VTE are venous stasis, vascular damage and hypercoaguability. Predisposing factors include reduced mobility, medical co-morbidities, surgery, active cancer, previous VTE, drugs, pregnancy, central lines, thrombophilias, obesity and varicose veins. In approximately $20 \%$ idiopathic PE may occur.

Karen KK Sheares, respiratory consultant, Pulmonary Vascular Diseases Unit, Papworth Hospital NHS Foundation Trust, Cambridge

\section{Symptoms and signs}

The symptoms include dyspnoea, pleuritic or retrosternal chest pain, cough, haemoptysis and syncope. Clinically the patient may be tachypnoeic, tachycardic, and hypoxic with an elevated jugular venous pressure, a gallop rhythm, a widely split second heart sound, a tricuspid regurgitation murmur and possible flow murmurs around the embolic obstructions. With pulmonary infarction there may be a pleural rub and pyrexia. In severe cases, the right ventricle fails to cope with the increase in afterload resulting in systemic hypotension and shock. Signs of a DVT increase the index of suspicion for PE.

\section{Investigation of patients with suspected PE}

The symptoms and signs in acute $\mathrm{PE}$ are neither sensitive nor specific and only a third of referred patients will have PE. Hence a pre-test assessment of clinical probability should be undertaken in all cases. Several clinical predictive models based on risk factors, symptoms and signs have been developed. The Wells prediction model for $\mathrm{PE}$ has been validated and is the most widely used. ${ }^{5}$ As shown in Fig 1, PE can be excluded in patients who have a low clinical probability and a negative D-dimer without further investigations.

\section{Investigations}

Plasma D-dimer gives a measure of endogenous fibrinolysis. It has a good negative predictive value and is useful in excluding PE. It is not diagnostic as it has a low specificity and is elevated in a wide range of conditions including infection, trauma and cancer. It should not be measured in patients with a high clinical 